\title{
THE PREDICTION OF MOLECULAR CONFORMATION AS A BIOLOGICALLY SIGNIFICANT PROPERTY
}

\author{
LEMONT B. KIER \\ Massachusetts College of Pharmacy, Boston, Massachusetts, USA
}

\begin{abstract}
The value of molecular orbital theory in the hands of the chemist or biologist lies, hopefully, in its ability to derive information concerning the properties of molecules of interest to him, without complete reliance on the performance of an experiment. This expectation is becoming more of a reality as a result of work using all-valence electron semi-empirical molecular orbital methods to study conformations of drug molecules. Some of the work in our laboratory is described with notations as to the general agreement with experiment. The frequent insight into the pharmacophore definition is a major contribution of these studies. Finally, the implication for explanations of drug mechanisms and new drug design is present in each topic discussed.
\end{abstract}

\section{INTRODUCTION}

Chemical and physical events involving a drug at its critical point of action in the body are, at this time, obscure. We assume a specific target for many drug molecules and term this a receptor. If we acknowledge that a drug must be absorbed, transported, penetrate barriers and survive metabolism, we are left with the conclusion that rather specific structural features are necessary for a drug to efficaciously engage a receptor. These structural characteristics are basically (a) those atomic features suitable for the requisite drugreceptor interaction phenomena and (b) the appropriate spatial disposition of these features necessary to bring about the required simultaneous or required sequential interaction events with the receptor.

Since insight into the intricacies of receptor structure are currently denied us, we must examine molecules of high potency and therefore presumably of optimal interaction with receptors, to gain insight into the drug-receptor phenomena. If we can define what is essential in a molecule for optimal receptor interaction we can generalize as to what that receptor requires in a molecule (pharmacophore). We may further speculate on what may be the complementary binding features of a receptor. The generalization of a pharmacophore provides us with some rationale for the design of agonists and competitive antagonists.

The definition of electronic properties of a molecule, such as electron distribution, can frequently be accomplished in an approximate way from 
intuition. A knowledge of relative electronegativities of atoms in a molecule permits us to make relative assignments of charge excess or charge deficiency, in many cases.

A more complex problem arises when the identified pharmacophore is embedded in a molecule in which we cannot intuitively predict the shape or conformation. A very large number of drugs and agonists are molecules with at least one sigma bond and hence with at least one degree of doubt as to the structure in space. Indeed the estimation of conformation in many cases becomes the limiting factor separating us from a useful definition of a molecular structure.

We have known for some years that sigma or single bonds are neither free to rotate nor are they entirely rigid. The composition of conformational isomers at equilibrium, and the associated energy barriers are governed by a summation of all attractions and repulsions within the molecule. This mixture of patterns must then prevail in drug molecules in the vicinity of a receptor. How then can we hope to define what is the 'active' conformation at a receptor?

Over the years we have conducted our studies on drug molecule conformations with the operational hypothesis that the energetically preferred conformation was the 'active' conformation at the receptor ${ }^{1,2}$. This hypothesis has obviously been adopted by many investigators both before and since our earliest work. They have adopted the hypothesis since they have sought by various means to define what the preferred conformation is of many drug molecules, and have used their findings to explain or predict biological events.

It is very likely that intimate interaction between a drug and a receptor will mutually perturb both molecules so that new conformations will dominate, governed by the energetics of the new environment. It is our basic hypothesis however that at the most remote distance of drug-receptor engagement it is the preferred conformation of the drug or a conformation very close in energy to the preferred conformation which is 'recognized' by the receptor. If the Eorrect pharmacophore is present in the molecule at the time of 'recognition' then upon closer approach, the appropriate interactions will occur leading to an efficacious biological event. As a net gain in energy of interaction or bonding occurs, mutual perturbation will alter both species along energy-determined pathways, leading to either an efficacious effect if the pharmacophore is present or to an inactive affinity. This then is the hypothesis of remote recognition of preferred conformation.

We have found in several of our studies that when more than one potent agonist in a pharmacological class is analysed for preferred conformation, there are predicted or observed nearly identical patterns of essential features in the preferred conformations. This certainly suggests more than a coincidence. It reinforces our belief that the preferred conformation is a highly relevant structural parameter contributing to the drug's action. We interpret this as supporting the hypothesis of remote recognition of preferred conformation.

\section{EVALUATION OF CONFORMATION}

The prediction of the conformation of molecules has been of considerable interest to biological scientists for many years. Early approaches centred on 


\section{THE PREDICTION OF MOLECULAR CONFORMATION}

an intuition based on the presumed repulsive interaction of bulky groups across space. This gave rise to general rules of conformational preference which had some utility with small molecules. Unfortunately, this intuition was unable to perceive the attractive forces which are also a part of the total influence on conformation. Attractive forces are particularly prominent in heteroatom molecules and these are predominantly what the biological scientist encounters.

The advent of $x$-ray crystallography has permitted the mapping of the atoms of molecules in the solid state. The relevance of these conformations to solution phenomena is, however, obscure. In the crystal, the molecules are closely packed, interacting with each other and with gegenions if present. This is probably not the situation normally encountered in the dilute solutions of the biological milieux. Thus, biological conclusions derived from conformations deduced from $\mathrm{x}$-ray crystallographic analyses must always be considered in this light.

A more useful experimental approach to predicting conformation in a biological environment is through the use of n.m.r. analysis in water. This data, if properly analysed, gives a time-average conformation which can be of considerable value in subsequent biological interpretations. It is necessary, however, to actually have the compound under study and frequently the analysis of the n.m.r. data is extremely complex.

Other solution techniques for predicting preferred conformation include o.r.d., dipole movement and spectroscopic methods. Each is capable of giving useful, partial information on molecular conformation. Each, of course, requires that the compound be actually available for study.

\section{MOLECULAR ORBITAL PREDICTION OF CONFORMATION}

Another approach which has become available in the past decade is the use of all-valence electron, semi-empirical molecular orbital theory. This approximation of quantum mechanics makes it possible to calculate for fairly large molecules, a total energy behaving in an approximately parallel fashion to the true molecular energy. The consideration of all valence electrons makes this calculated total energy sensitive to the conformation of the molecule. Thus, energy minimization as a function of bond-angle variation is possible and the prediction of a preferred conformation is a consequence.

The first of these methods was developed by Hoffmann in $1963^{3}$, and is known as extended Hückel theory (EHT). Briefly, the method employs Hückel formalism; however, explicit consideration of non-bonded interactions and all overlap integrals are a refinement. Slater orbitals are employed and the computations require only one parameter, the valence state ionization potential for the Coulomb integral and indirectly for the resonance integral. The theory, the merits and evaluations of EHT have been reported ${ }^{4-6}$.

More recently, an all valence electron, semi-empirical molecular orbital theory known as the Complete Neglect of Differential Overlap (CNDO) has been proposed by Pople based on self-consistent field (SCF) formalism? ${ }^{7}$. Although this method employs a more sophisticated approximation of the wave function, it neglects differential overlap. 
Comparisons of these two methods reveal their relative strengths and shortcoming ${ }^{4}$. In general, the CNDO method is superior for charges, EHT predicting greatly exaggerated values. The major value of EHT lies in its ability to correctly predict the preferred conformation. This has been demonstrated for numerous hydrocarbons ${ }^{3}$ and more recently for a wide variety of heteroatom molecules ${ }^{1,2}$.

A large number of molecular orbital predictions of biological molecule conformations have been accomplished using EHT. The record of agreement between calculated and experimental values has been good. A significant amount of useful information has emerged from these predictions pertinent to the structure-activity relationships, the consideration of molecular mechanisms, and the rationale for new drug design ${ }^{1,2}$.

\section{MUSCARINIC AGENTS}

The first MO study of the conformation of a neurotransmitter was reported on acetylcholine ${ }^{8}$. The prediction of a gauche chain is in agreement with an n.m.r. analysis ${ }^{9}$. In the same study, the prediction of the conformations of the muscarinic agonists muscarine and muscarone were reported. Both predictions have been supported by $\mathrm{x}$-ray analyses ${ }^{10,11}$. This early work led to the encouraging belief that EHT calculations of conformation could mirror physical reality. The study also revealed that all three muscarinic agonists in their predicted preferred conformations resulted in the virtual superpositioning of the heteroatoms. This led to the prediction that this pattern of the heteroatoms was the muscarinic pharmacophore, Figure 1. It is noteworthy that this pharmacophore is practically identical to the muscarinic pharmacophore derived from extensive structure activity

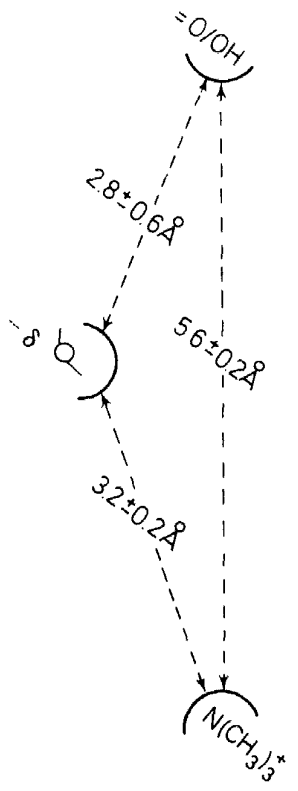

Figure 1. Predicted muscarinic pharmacophore ${ }^{8}$ 
studies $^{12}$. The findings support the hypothesis that the preferred conformation is relevant to this series of drug-receptor events.

Similar calculations on oxotremorine, a CNS muscarine agent, revealed a pattern of structural features compatable with Figure $1^{13}$.

The early success of these studies both in their agreement with experiment and in the biological significance of the interpretations, have resulted in the continued use of this method and general approach ${ }^{1,2}$.

\section{NICOTINIC AGENTS}

In a companion study to the muscarinic agents, calculations were performed on the nicotinic agent nicotine ${ }^{14}$. Two equivalent conformations were found in agreement with n.m.r. data ${ }^{15}$. In one of these conformers there was found a distance separating the pyridyl nitrogen and onium group, comparable to the carbonyl oxygen-onium group distance in a conformer of acetylcholine. This pattern was proposed as the nicotinic pharmacophore,

$$
\mathrm{X}^{-} \longrightarrow \sim 4.9 \AA \longrightarrow(\mathrm{N})^{+}
$$

Figure 2. Predicted nicotinic pharmacophore ${ }^{14}$

Figure 2 and that conformer of acetylcholine proposed as being the nicotinic form of acetylcholine ${ }^{14}$. MO-calculated conformations of other potent nicotinic agents such as phenylcholine ether ${ }^{16}$ and neostigmine ${ }^{17}$ support this pharmacophore hypothesis. The prediction of the phenylcholine ether conformation is in agreement with the crystal conformation of xylocholine ${ }^{18}$.

As a result of these studies, the conclusion was reached that acetylcholine exerts two actions, muscarinic and nicotinic, by engaging two different receptors with two different pharmacophores. The two pharmacophores are constituted from different sets of functional features in different conformations of the molecule ${ }^{2}$.

\section{HISTAMINE}

Molecular orbital calculations have led to the prediction of two equivalent conformations of histamine with gauche and trans side chains respectively ${ }^{19}$.

This prediction has been verified by n.m.r. analysis ${ }^{20}$. A CNDO-type calculation predicts only a gauche conformation as preferred ${ }^{21}$.

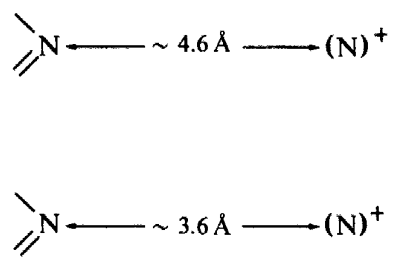

Figure 3. Predicted histamine pharmacophores ${ }^{19}$ 
On the basis of the prediction of the two conformations, the hypothesis was proposed that the dual action of histamine was due to the engagement of one receptor, designated $\mathrm{H}_{1}$, with the trans conformer and the second receptor, the acid secretory receptor $\mathrm{H}_{2}$, with the gauche conformer ${ }^{19}$, Figure 3. This is then a parallel of the acetylcholine dual-action, dualconformation hypothesis ${ }^{2,8,14}$.

\section{SEROTONIN}

The preferred conformation of serotonin was predicted, using EHT, to have a trans side chain ${ }^{22}$. Modified $\mathrm{CNDO}^{23}$ and $\mathrm{INDO}^{24}$ calculations have predicted a gauche to a cis preference for the side chain. Recent n.m.r. analysis reveals a trans preference ${ }^{25}$, in agreement with the EHT calculation.

A serotonergic pharmacophore was postulated on the basis of the predicted conformation $^{22}$, Figure 4 . It is interesting to note that the inter-nitrogen

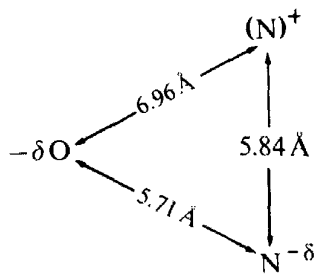

Figure 4. Predicted serotonin pharmacophore 22

distance in this pharmacophore is mimicked in the lysergic acid diethylamide (LSD) molecule, a competitive antagonist. Another similarity has been commented upon, relating the serotonin pharmacophore and the active metabolite, 11 -hydroxy- $\Delta^{9}$-tetrahydrocannabinol ${ }^{26}$. This could be a significant relationship in the hallucinogenic action of this molecule and LSD.

\section{PREGNANE STEROIDS}

The side chain conformations of progesterone, corticosterone and cortisol were predicted using EHT-MO ${ }^{27}$. All three side chains were predicted to form a plane with the $17 \alpha$ substituent. The prediction of the progesterone side chain conformation is within $30^{\circ}$ of a solution dipole moment study ${ }^{28}$. The predictions of the corticosterone and cortisol conformations are in close agreement with experimental results from infrared and n.m.r. studies $^{29,30}$.

Portions of the predicted cortisol pattern of charged atoms, Figure 5, were observed to be comparable to charged patterns for either histamine ${ }^{19}$ or serotonin $^{22}$. Since these two amines have been implicated as being inflammagenic $^{31}$ and cortisol is a potent anti-flammatory agent ${ }^{32}$, it was postulated that cortisol might evoke this action by an interaction with either or both histamine and serotonin receptors by virtue of these common structural features.

The hypothesis has received some experimental support from the recent observation that cortisol is effective in competing for histamine-binding sites 


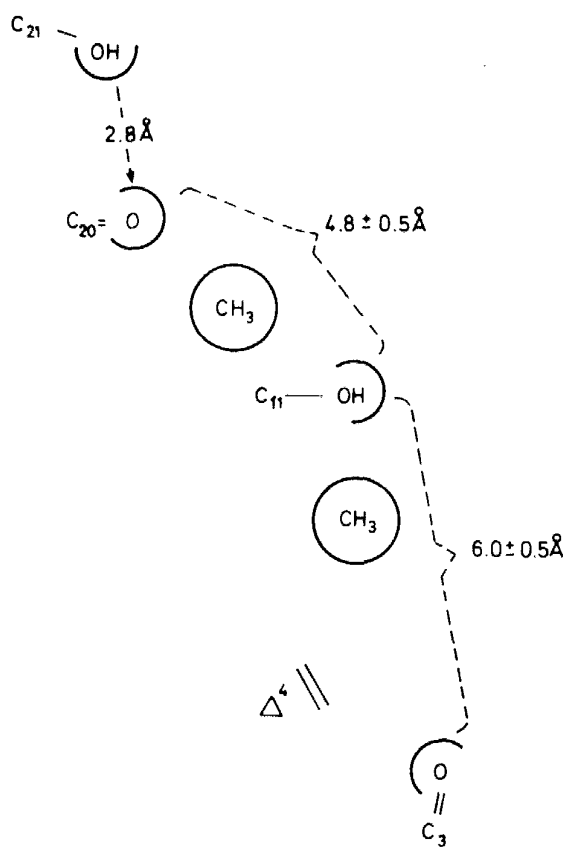

Figure 5. Predicted cortisol pattern ${ }^{27}$

on biopolymers ${ }^{33}$. A subsequent EHT-MO study involving non-steroidal anti-inflammatory agents including indomethacin confirmed that the common structural patterns predicted, prevailed in these drugs ${ }^{34}$. The predicted conformation of indomethacin is in agreement with the reported crystal structure of this molecule ${ }^{35}$. It is interesting to contemplate the roles of serotonin as a platelet aggregation promoter and the common antiinflammatory agents as platelet aggregation inhibitors as a possible parallel of this hypothesis of anti-inflammatory activity.

\section{ADRENERGIC AGENTS}

EHT-MO predictions of the adrenergic agents ephedrine and pseudoephedrine have been reported ${ }^{36}$. The conformation predicted for ephedrine is in agreement with n.m.r. analysis ${ }^{37,38}$; however, the prediction of the pseudoephedrine conformation agrees only with a minor contributor to the solution equilibrium ${ }^{38}$. The predicted conformation for the $\alpha$-adrenergic agonist, norepinephrine, presents the same pharmacophore ${ }^{39}$, Figure 6. This prediction is in agreement with the crystal conformation ${ }^{40}$. Other studies using the INDO ${ }^{41}$ and CNDO methods ${ }^{42}$ predict the coexistence of a trans and a gauche conformation.

It is well known that an unsubstituted catecholamine such as norepinephrine or a monomethyl derivative like epinephrine is predominantly $\alpha$ adrenergic. Increasing the bulk of the mono-alkyl substituent in this series 


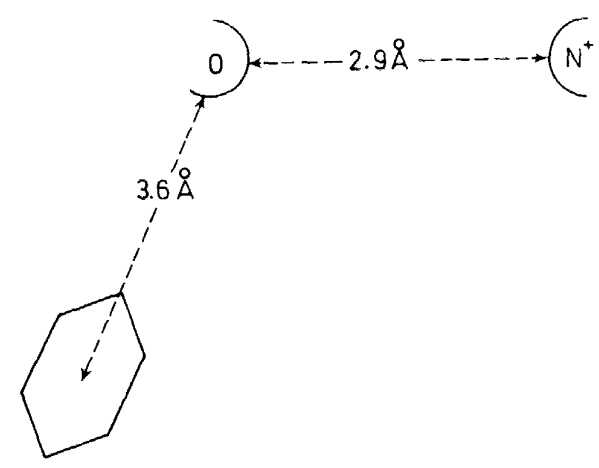

Figure 6. Predicted $\alpha$-adrenergic pharmacophore ${ }^{36,39}$

increases $\beta$-adrenergic activity while at the same time $\alpha$-adrenergic activity is obliterated. Thus, isopropyl norepinephrine (isoproteranol) is a standard for almost pure $\beta$-adrenergic activity.

Two theories have been proposed to explain this reversing pair of trends in the catecholamine series. One theory proposed that the increasing bulk of the $\mathrm{N}$-substituent increases the barrier to rotation between the methylene groups so that the ease of assuming a gauche conformation will be a function of the $\mathrm{N}$-substituent bulk ${ }^{\mathbf{4 3}}$. The theory then proposes that the different conformers, gauche and trans, influence the occurrence of two different reactions, each characteristic of the $\alpha$ and $\beta$ adrenergic receptor. The second theory proposes that the $\mathrm{N}$-substituent influences the charge on the onium group which influences the reactivity to one or the other adrenergic receptor ${ }^{44}$.

A recent study employing EHT for conformation and CNDO and $a b$ initio calculations for charge densities has been reported on the catecholamine series norepinephrine, epinephrine, $N$-ethylnorepinephrine and isoproteranol $^{45}$. These studies revealed no predicted change in the trans preference for any of the series. Further, they showed an almost identical energy of the barrier from a trans to a gauche conformation for all members of the series. These results argue against the theory of variable flexibility of the methylenemethylene bond ${ }^{43}$. Charge densities were calculated on simulated onium systems using both CNDO and $a b$ initio methods ${ }^{45}$. These results revealed no appreciable change in onium charge in the series, which is at variance with the charge theory ${ }^{44}$.

An alternative theory was proposed based on these calculations. It was postulated that $\alpha$-adrenergic activity requires an onium hydrogen atom, probably as a hydrogen bond donor. The $\beta$-adrenergic receptor, however, was postulated to require an alkyl group at the $\mathrm{N}$-substituent position. It was proposed that this $\mathrm{N}$-substituent was involved in a dispersion interaction with the receptor and was optimal when there was a branched hydrocarbon such as an isopropyl group. These authors raised the intriguing suggestion that the onium group has a constant influence or was perhaps not essential for $\beta$-adrenergic activity and that a methylene group could replace it. It was noted that such a compound has recently been made and reported ${ }^{46}$. This 


\section{THE PREDICTION OF MOLECULAR CONFORMATION}

compound, the methylene analogue of isoproteranol, was found to have modest $\beta$-adrenergic activity. A parallel was also drawn between this hypothesis and the structure and $\beta$-adrenergic activity of prostaglandin $E_{1}^{45}$.

\section{DOPAMINE}

An EHT calculation on dopamine, holding the ring hydroxyls out of the ring plane predicted a gauche conformation ${ }^{47}$. A calculation apparently on just the side chain conformation predicted a trans conformation ${ }^{48}$. A recalculation of the molecule, holding the hydroxyls in the ring plane, predicts a trans conformation ${ }^{26}$. Crystal studies indicate a trans preference for the salt ${ }^{49}$ while n.m.r. analysis indicates a contribution from both isomers ${ }^{48}$. It has been suggested that the gauche conformer of dopamine might account for its ability to interact with a receptor different from that of noradrenalin ${ }^{47}$.

An interesting structural similarity can be observed between dopamine and the morphine-like analgesics. A basic nitrogen atom can be found, in all of these compounds, about $4 \AA$ from the centre of a phenyl ring. This is a dimension found in the gauche form of dopamine ${ }^{47}$. It is possible that the mechanism of analgesia involves dopamine with the analgesics acting as inhibitors at a specific site.

\section{CENTRAL INHIBITORY TRANSMITTERS}

Calculations on the conformation of $\gamma$-aminobutyric acid (GABA) and an agonist, muscimol, have revealed a common pattern of charged structural features arising from a trans conformation of GABA, leading to the prediction of a pharmacophore ${ }^{50}$. Support for this prediction can be found in the fact that the structurally rigid acetylenic analogue aminotetrolic acid is a GABA agonist ${ }^{51}$.

Further support is found in the prediction of a conformation for the competitive antagonist bicuculline, in which the hypothetical pharmacophore for GABA is reproduced ${ }^{52}$. Calculations on the agonist $\beta$-hydroxy GABA indicate a gauche and a trans preference ${ }^{52}$.

In contrast to GABA, the centrally-acting inhibitory transmitter glycine is predicted to have a conformation not resembling GABA in its salient structural features ${ }^{52}$. This supports the belief that these two agents are acting at separate receptors.

\section{PROSTAGLANDIN}

Prostaglandin E-1 has recently been studied using EHT for some conformational properties, iterative EHT for atom charges and a method using monopole-bond polarizabilities for the calculation of the interaction energies between the side chains, contributing to the conformation ${ }^{53}$. The calculations predicted a graded series of preferences for the side chains, the most prominent of which involve an intimate interaction and mutual fixation of the side chain.

The dispersion forces are significant, exceeding $2 \mathrm{kcal} \mathrm{mole}^{-1}$ in the most preferred conformer. These predictions are consistent with a recent $\mathrm{x}$-ray 
study which reveals one of the most prominent of the predicted conformers ${ }^{54}$. Structural rigidity imparted by the side chain interactions thus appears to be an essential feature in these molecules. These predictions may afford a means of studying the influence of side chain modifications and their influence on the diverse actions of these molecules.

\section{PEPTIDE HORMONES}

Following the initial predictions of amino acid conformations using EHT in $1969^{55-57}$ a number of studies have been reported on all-valence electron MO methods in this area (recently reviewed ${ }^{26}$ ). There is general agreement found between prediction and available experimental evidence. Two studies have been reported on the conformations of small polypeptide hormones. These have been predicted from the individual residue predictions for bradykinin $^{58}$ and gastrin ${ }^{59}$. Hopefully these studies can point the way toward the very desirable goal of predicting small polypeptide conformation.

\section{SWEET TASTE PHARMACOPHORE}

At the present time it is believed that structural features imparting a sweet taste to a molecule include an atom capable of accepting a hydrogen bond (B) and a structure feature with a polar hydrogen $(\mathrm{A}-\mathrm{H})$, Figure $7^{60}$. The presence of this duo, $\mathrm{A}-\mathrm{H}$ and $\mathrm{B}$, can be found in a widely diverse group of

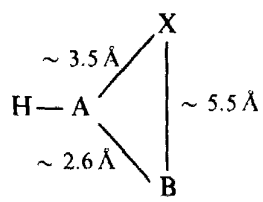

Figure 7. Predicted sweet taste pharmacophore or glucophore ${ }^{62}$

sweet-tasting molecules. A number of observations, however, suggest that this pharmacophore (or glucophore) may be incomplete. The fact that several D- amino acids are sweet while their L- isomers are bitter ${ }^{61}$ is evidence of stereoselectivity, and a third receptor binding site.

EHT calculations on these amino acids has identified a common third molecular feature, assuming the $\mathrm{A}-\mathrm{H}, \mathrm{B}$ system to be $\mathrm{N}-\mathrm{H}$ and an oxygen atom $^{62}$. This has led to the postulation of a glucophore, Figure 7 , with the third feature being an atom or group capable of dispersion bonding to a receptor feature.

Examination of a wide variety of sweet molecules of high potency has revealed the presence of this glucophore. Further evidence for dispersion bonding being involved at the third site of the glucophore is furnished by the finding of a good correlation between a group polarizability parameter and the sweetness level in a series of substituted nitroanilines ${ }^{62}$. 


\section{CONCLUSION}

With the passage of time and the contributions from our laboratory and others, we are strengthened in our view that MO theory can make a contribution to our understanding of drug molecule structure.

If the investigator is constantly aware of the nature of his semi-empirical methods, their strengths and limitations he can be guided into meaningful utilization of these methods. If he fully acquaints himself with the nature of biological events and how theory can explain or predict these events, meaningful applications can be made.

From the predictions made in our studies and the structure-activity hypotheses drawn, the medicinal chemist and the molecular pharmacologist have perhaps a new rationale for compound design or new relationships to derive mechanisms.

The validity of these hypotheses await further support. However, as is usually the case in scientific endeavour, the real scarcity is in new ideas. We feel that molecular orbital theory is a rich source for their derivation.

\section{REFERENCES}

1 L. B. Kier, Molecular Orbital Theory in Drug Research, Academic Press, New York (1971).

${ }^{2}$ L. B. Kicr in Fundamental Concepts in Drug-Receptor Interactions (J. F. Danielli, J. F. Moran and D. J. Triggle, eds.) Academic Press, New York (1970).

3 R. Hoffman, J. Chem. Phys. 39, 1397 (1963).

4 J. R. Hoyland in Molecular Orbital Studies in Chemical Pharmacology, (L. B. Kier, ed.) Springer-Vorlag, New York (1970).

${ }^{5}$ L. C. Allen and J. D. Russell, J. Chem. Phys. 46, 1029 (1967).

6 G. Blyholder and C. A. Coulson, Theoret. Chim. Acta 40, 316 (1968).

7 J. A. Pople, D. P. Santry and G. A. Segal, J. Chem. Phys. 43, 5129 (1965),

${ }^{8}$ L. B. Kier, Mol. Pharmacol. 3, 487 (1967).

9 C. Culvenor and N. Ham, Chem. Commun. 537 (1966).

${ }_{10}$ F. Jellinek, Acta Cryst. 10, 277 (1957).

11 P. Pauling and T. J. Petcher, Nature New Biol. 236, 112 (1972).

12 A. H. Beckett, N. J. Harper and J. W. Clitherow, J. Pharm. Parmacol. 15, 362 (1963).

13 L. B. Kier, J. Pharm. Sci. 59, 112 (1970).

14 L. B. Kicr, Mol. Pharmacol. 4, 70 (1968).

15 T. R. Simpson, J. C. Craig and W. D. Kumler, J. Pharm. Sci. 56, 708 (1967).

${ }^{6}$ L. B. Kier, J. Med. Chem. 14, 80 (1971).

7 J. W. Crow and W. C. Holland, J. Med. Chem. 15, 429 (1972).

18 P. Coggon, A. T. McPhail and A. M. Roe, Nature, 224, 1200 (1969).

19 L. B. Kier, J. Med. Chem. 11, 441 (1968).

20 A. F. Casy, R. R. Ison and N. S. Ham, Chem. Commun. 1343 (1970).

21 J. L. Coubeils, P. Courriere and B. Pullman, Compt. Rend. Acad. Sci., Paris 272, 1813 (1971).

L. B. Kier, J. Pharm. Sci. 57, 1188 (1968).

P. Courriere, J. L. Coubeils and B. Pullman, Compt. Rend. Acad. Sci., Paris, 272, 1697 (1971).

S. Kang and M. H. Cho, Theoret. Chim. Acta 22, 176 (1971).

25 R. R. Ison, P. Partington and G. C. K. Roberts, J. Pharm. Pharmacol. 24, 84 (1972).

26 L. B. Kier in Advances in Chemistry, 114, 278 (1972).

7 L. B. Kier, J. Med. Chem. 11, 915 (1968).

28 N. L. Allinger and M. A. DaRooge, J. Am. Chem. Soc. 83, 4256 (1961).

29 W. G. Cole and D. H. Williams, J. Chem. Soc. 1849 (1968).

30 W. G. Cole and D. H. Williams, J. Chem. Soc. 748 (1970).

31 W. G. Spector and D. A. Willoughby, Ann. N.Y. Acad. Sci. 116, 839 (1964).

32 C. A. Winter, Progr. Drug Res. 10, 139 (1966).

${ }^{33}$ C. Botre, M. Marchetti, M. Del Vecchio, C. Lionetti and A. Memoli, J. Med. Chem. 12, 832 (1969). 


\section{LEMONT B. KIER}

34 L. B. Kier and M. W. Whitehouse, J. Pharm. Pharmacol. 20, 793 (1968).

35 T. J. Kistenmachen and R. E. Marsh, J. Am. Chem. Soc. 94, 1340 (1972).

36 L. B. Kier, J. Pharmacol. Exptl. Therap. 164, 75 (1968).

37 J. B. Hyne, Can. J. Chem. 39, 2536 (1961).

38 P. Portoghese, J. Med. Chem. 10, 1057 (1967).

39 L. B. Kier, J. Pharm. Pharmacol. 21, 93 (1969).

40 D. Carlstrom and R. Bergin, Acta Cryst. 23, 313 (1967).

41 L. Pedersen, R. E. Hoskins and H. Cable, J. Pharm. Pharmacol. 23, 216 (1971).

42 B. Pullman, J. L. Coubeils, P. Courriere and J. P. Gervois, J. Med. Chem. 15, 17 (1972).

43 A. A. Larsen, Nature 224, 25 (1969).

44 P. Pratesi and E. Grava, Adv. Drug Res. 2, 127 (1965).

45 J. M. George, L. B. Kier and J. R. Hoyland, Mol. Pharmacol. 7, 328 (1971).

${ }^{46}$ L. Villa, V. Ferri, E. Grana and O. Mastelli, Il Farmaco, Ed. Sci. 25, 118 (1970).

47 L. B. Kier and E. B. Truitt, J. Pharm. Exptl. Therap. 174, 94 (1970).

48 T. M. Bustard and R. S. Egan, Tetrahedron 27, 4457 (1971).

49 R. Bergin and D. Carlstrom, Acta Cryst. B24, 1506 (1968).

50 L. B. Kier and E. B. Truitt, Experientia 26, 988 (1970).

${ }^{51}$ P. M. Beart, D. R. Curtis and G. A. R. Johnston, Nature New Biol. 234, 80 (1971).

52 L. B. Kier, Experientia, in press.

53 J. R. Hoyland and L. B. Kier, J. Med. Chem. 15, 84 (1972).

54 I. Rabinowitz, P. Ramwell and P. Davison, Nature New Biol. 223, 88 (1971).

55 R. Hoffman and A. Imamura, Biopolymers 7, 207 (1969).

${ }^{56}$ L. B. Kier and J. M. George, Theoret. Chim. Acta 14, 258 (1969).

57 A. Rossi, C. W. David and R. Schor, Theoret. Chim. Acta 14, 429 (1969).

58 L. B. Kier and J. M. George in Molecular Orbital Studies in Chemical Pharmacology, (L. B. Kier, ed.) Springer-Verlag, New York (1970).

59 L. B. Kier and J. M. George, J. Med. Chem. 15, 384 (1972).

60 R. S. Shallenberger and T. E. Acree, Nature 216, 480 (1967).

61 J. Solms, J. Agr. Food Chem. 17, 686 (1969).

${ }^{62}$ L. B. Kier, J. Pharm. Sci. 61, 1394 (1972). 